\title{
Analytic performance evaluation of opportunistic spectrum access with detection errors
}

\author{
Patrick Tortelier \\ Orange Labs Issy les Moulineaux, France \\ patrick.tortelier@orange-ftgroup.com
}

\begin{abstract}
This paper aims at giving some results on the performances of opportunistic spectrum access when taking into account sensing errors: non detection, which is the cause of collisions with a transmitting user, and false alarm, which is a cause of missed opportunity to access the channel. We give the probability $\mathcal{A}_{n}(i, j)$ of having $i$ collisions and $j$ successful access to the channel within $n$ time slots when the primary user's activity can be described by a two-state On/Off model. Our derivation allows us to take into account aggressive or conservative policy, that is to say the secondary user does not systematically obey the indication of the sensing stage, but with a given probability because the sensing is known to be prone to either false alarm or non detection with high probability.
\end{abstract}

\section{General Terms}

Theory

\section{Keywords}

Opportunistic spectrum access, sensing errors, performance evaluation

\section{INTRODUCTION}

In a growing number of papers we can see the emergence of several new schemes of spectrum access modes departing from the Command and Control scheme which is the way spectrum use is regulated today. These schemes are intended to provide a better use of radio spectrum than the Command and Control scheme, which is viewed as too static, by bringing in some flexibility in the way users access to the spectrum. These new schemes range from exclusive use (there is always only one licensed user in a frequency band, but its license is temporary), to shared use of a primary licensed spectrum (either underlay or opportunistic) to commons (unlicensed spectrum, as ISM band at $2.4 \mathrm{GHz}$ or UNII bands at $5 \mathrm{GHz}$ ). For a comprehensive classification the reader is referred to $[1,2]$.

Permission to make digital or hard copies of all or part of this work for personal or classroom use is granted without fee provided that copies are not made or distributed for profit or commercial advantage and that copies bear this notice and the full citation on the first page. To copy otherwise, to republish, to post on servers or to redistribute to lists, requires prior specific permission and/or a fee. VALUETOOLS 2011, May 16-20, Paris, France Copyright (c) 2011 ICST 978-1-936968-09-1 DOI 10.4108/icst.valuetools.2011.245613
Among these schemes, opportunistic access has gained a lot of interest and has become quite synonymous to Cognitive Radio (CR). This is a scenario where a secondary user can share a primary user's spectrum when it is not transmitting, without coordination. This last point is important, it means there is no beacon advertising that the channel is free or that primary user (PU) is going to transmit again, so that the secondary user (SU) must implement some sensing method to detect idle periods (if any) of the primary user and (more important) to detect when the PU is transmitting again. For a CR oriented presentation of sensing the reader is referred to $[3,4]$, and with a more signal processing emphasis to [5].

Sensing schemes are not perfect, especially when the decision must be taken quickly. It may happen that the secondary user (SU) decides that the channel is occupied while it is free (this is a false alarm and, as a result, a missed opportunity to use the channel) or that the channel is empty while there is actually someone (a non detection, and a collision with the already present user). These two events have respective probabilities denoted $P_{F A}$ (false alarm probability) and $P_{N D}$ (non detection probability) which are essential parameters of the quality of the detection scheme, and can be used subsequently for various analytical performance computations.

These two probabilities are not independant : for a given signal to noise ratio the possible pairs $\left(1-P_{N D}, P_{F A}\right)$ lie on a Receiver Operating Characteristic (ROC) curve $[6,3]$ such that we cannot have simultaneously small non detection and false alarm probabilities as depicted on a typical ROC curve in fig. 1: $P_{N D} \rightarrow 0 \Rightarrow P_{F A} \rightarrow 1$. The choice of the functionning point is thus the result of some trade-off.

This paper aims at giving an analytical evaluation of what can be achieved for a secondary user (in terms of successful access to the spectrum resource) when taking into account the impairments of the sensing (false alarm and non detection probabilities) and under the assumption that the occupancy of each frequency band can be described by an On/Off model as sketched in section 2. We also show how to introduce some kind of policy in the behaviour of the secondary user with respect to the sensing impairments.

\section{SIMPLE ON/OFF MODEL}

Reference [7] reports extensive measurements of time and frequency occupancy of spectrum frequency bands. Time occupancy is described therein as a binary information (On/Off) 


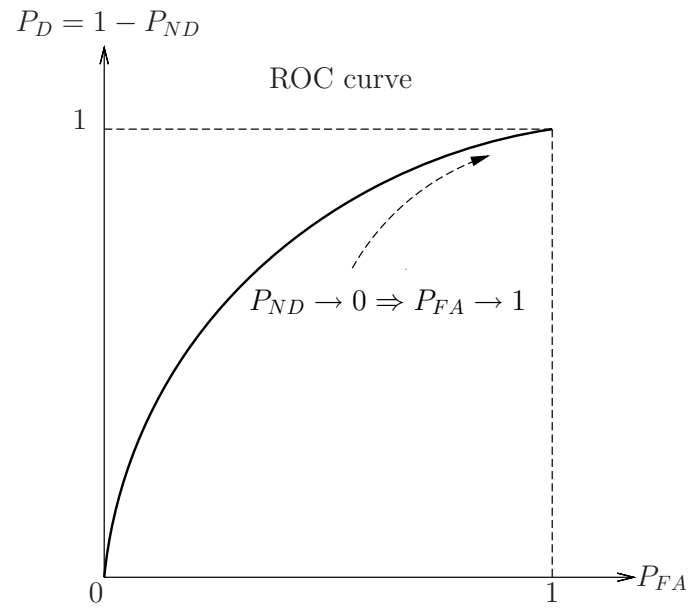

Figure 1: A typical ROC curve

and results of section 4.2 of this paper, pertaining to the Run and Burst distributions, show that they are accurately fitted by geometric or lognormal distributions. A geometric distribution of runs and bursts is the result of a time occupancy modelled as a two state On/Off (or Transmit/Idle) Markov model that will be used throughout the present paper.

The remainder of this section is devoted to a review of some simple results that can be useful when the primary user traffic is such a two state On/Off process, especially if we adopt the secondary user point of view (what are the performances that can be expected for the secondary user). In this relatively simple model we assume that the whole frequency band of interest is divided in a number $\nu$ of sub-bands of respective widths $B_{1}, B_{2}, \ldots B_{\nu}$. Each sub-band is either occupied (the primary user is transmitting) or idle during each time slot $\Delta T$ as sketched in fig. 2. The time vary-

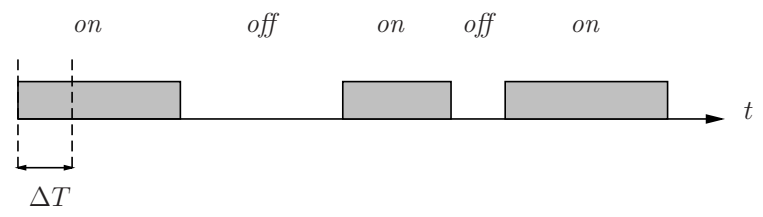

Figure 2: Time occupancy of a given sub-channel $B_{i}$

ing activity of a given sub-band is modelled by a two state model: state On if the band is occupied and Off if nobody transmits in the band during the slot under consideration. With these notations the time activity of a given sub-band $B_{i}$ can be modelled by a two-state Gilbert model with given transition probabilities between states (fig. 3).

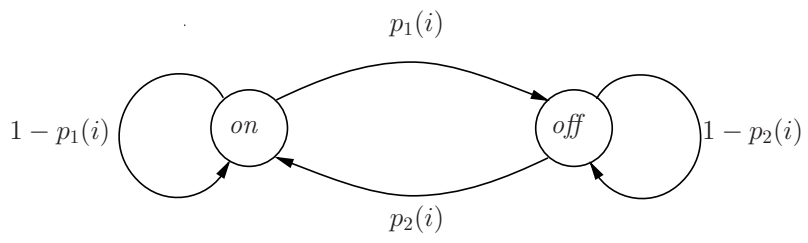

Figure 3: Simple On/Off model for channel $i$
We denote $p_{1}(i)$ the transition probability from state On to state Off for the sub-band $B_{i}$, conversely $p_{2}(i)$ is the transition probability from state Off to state On. The steady state probabilities $P_{o n}, P_{o f f}$ of this sub-band to be active or idle are then given by the classical formula $[8,9]$ :

$$
P_{\text {on }}(i)=\frac{p_{2}(i)}{p_{1}(i)+p_{2}(i)}, \quad P_{o f f}(i)=\frac{p_{1}(i)}{p_{1}(i)+p_{2}(i)}
$$

From this simple setting we can obtain analytical statistics. For instance we can derive the number of active sub-bands in a given time slot $\Delta T$ by use of the polynomial

$$
P(X)=\prod_{i=1}^{\nu}\left(P_{o f f}(i)+P_{o n}(i) X\right)=\sum_{j=0}^{\nu} A_{j}^{(\nu)} X^{j}
$$

The coefficient $A_{j}^{(\nu)}$ is the probability of having $j$ active subbands and $\nu-j$ idle ones. With this polynomial we can, for instance, compute the average number of active sub-bands in a time slot. Indeed, with the substitution $X=1$ in the derivative $P^{\prime}(X)$ we obtain this average value as:

$$
P^{\prime}(1)=\sum_{j=0}^{\nu} j A_{j}^{(\nu)}
$$

Furthermore, as $P(X)$ is a product of (simple) polynomials we can also use its logarithmic derivative to write:

$$
\begin{aligned}
\frac{P^{\prime}(X)}{P(X)} & =\sum_{i=1}^{\nu} \frac{P_{o n}(i)}{P_{o f f}(i)+X P_{o n}(i)} \\
\frac{P^{\prime}(1)}{P(1)} & =\sum_{i=1}^{\nu} \frac{P_{o n}(i)}{P_{o f f}(i)+P_{o n}(i)}=\sum_{i=1}^{\nu} P_{o n}(i)
\end{aligned}
$$

so that, finally, using $P(1)=1$, we do not have to compute the $A_{j}^{(\nu)}$ coefficients to obtain the average number of active sub-bands:

$$
P^{\prime}(1)=\sum_{i=1}^{(\nu)} \frac{p_{2}(i)}{p_{1}(i)+p_{2}(i)}
$$

With the assumption of equal width sub-bands (2) is an indicator of the activity of a given frequency-band, and, as a consequence, of the available room for a secondary access. If two frequency bands are considered for a possible opportunistic access the SU will choose the frequency band with the lower average number of active sub-bands, because it means there are more opportunities to access the channel. That can be useful in case of limited resources because the sensing is restricted to the most promizing frequency-band.

\section{STATISTICS FOR A GIVEN SUB-BAND}

The same approach can provide interesting results when considering a single sub-band $B_{i}$ over a number $n$ of successive time slots $\Delta T$. To this end we introduce some quantities: $A(m, n)$ is the probability of having $m$ active slots among $n$ when the initial state is On.

Likewise, $B(m, n)$ is the same probability when the initial state is Off.

These probabilities can be computed iteratively as sketched in fig. 4 for the recurrence on the $A(m, n)$; we set the initial state to On, and make the distinction between the next $O n$ or Off state in order to make apparent the first state transition so that $A(m, n)$ can be expressed with $A(m-1, n-1)$ 
and $B(m-1, n-1)$ because, the first time slot being On, it remains only $m-1$ active slots among the last $n-1$ time slots.

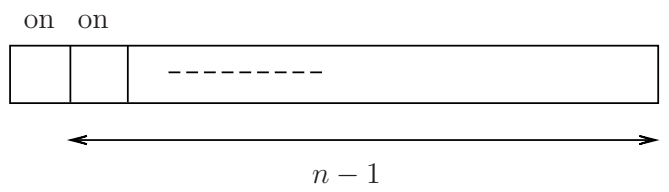

on off

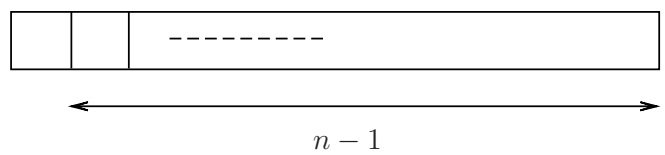

Figure 4: recurrence for the computation of $A(m, n)$

$$
A(m, n)=\left(1-p_{1}\right) A(m-1, n-1)+p_{1} B(m-1, n-1)
$$

Likewise, the same reasoning allows us to make apparent that, when the initial state is Off, the $m$ active time-slots must be located within the remaining time-slots, so that we have the following recurrence:

$$
B(m, n)=p_{2} A(m, n-1)+\left(1-p_{2}\right) B(m, n-1)
$$

The initial conditions for $(3 \mathrm{a})$ and $(3 \mathrm{~b})$ are obvious: $A(0,1)=$ $0, A(1,1)=1$ and $B(0,1)=1, B(1,1)=0$.

Averaging these probabilities over the steady state probabilities of on and off states give the unconditional probability $P(m, n)$ of having $m$ occupied slots over $n$ consecutive slots:

$$
P(m, n)=P_{o n} \times A(m, n)+P_{o f f} \times B(m, n)
$$

We can gather these quantities as coefficients of polynomials:

$$
\begin{aligned}
& A_{n}(X)=\sum_{m=0}^{n} A(m, n) X^{m} \\
& B_{n}(X)=\sum_{m=0}^{n} B(m, n) X^{m} \\
& P_{n}(X)=\sum_{m=0}^{n} P(m, n) X^{m}=P_{o n} A_{n}(X)+P_{o f f} B_{n}(X)
\end{aligned}
$$

The recurrences (3a) and (3b) can now be written as easy to implement recurrences on polynomials:

$$
\begin{aligned}
& A_{n}(X)=\left(1-p_{1}\right) X A_{n-1}(X)+p_{1} X B_{n-1}(X) \\
& B_{n}(X)=p_{2} A_{n-1}(X)+\left(1-p_{2}\right) B_{n-1}(X)
\end{aligned}
$$

with the initial conditions $A_{1}(X)=X$ and $B_{1}(X)=1$.

\subsection{Comparison with the binomial distribution}

The distribution $P(m, n)$ is generally (very) different from a binomial distribution on $n$ trials with the active slot probability $P_{o n}$, that is $P^{\prime}(m, n)=\left(\begin{array}{c}n \\ m\end{array}\right) P_{o n}^{m} \times\left(1-P_{o n}\right)^{n-m}$, as depicted in fig. 5 for $n=100$ time slots and transition probabilities $p_{1}=0.2, p_{2}=0.1$. This basic example is an indication of the potential interest of the On/Off model to model the time occupancy of a frequency band. Nevertheless it does not give any indication of the way the occupied states are distributed: are they completely sparse or are they rather clustered with long idle periods between them. A burst is defined a consecutive series of occupied states

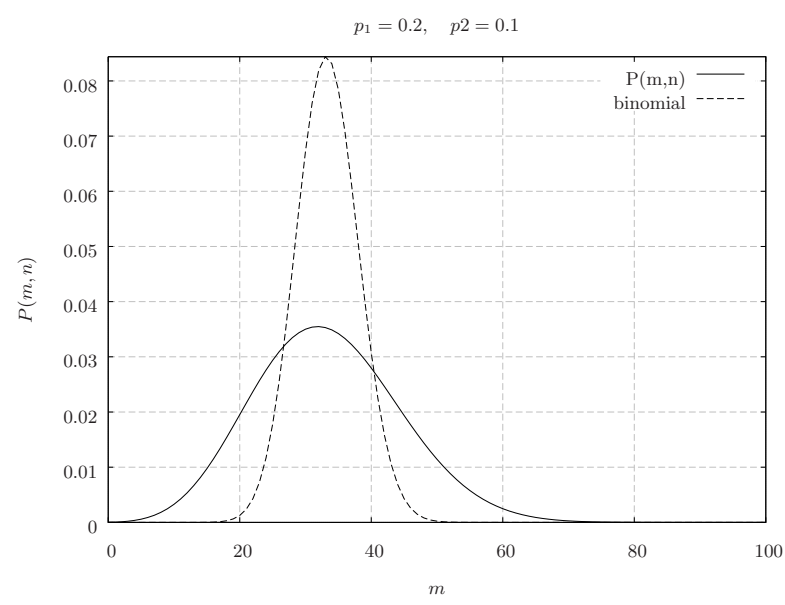

Figure 5: Comparison of the distribution $P(m, n)$ and the binomial distribution with parameter $P_{o n}$

without idle states so that the channel cannot be accessed within the burst. Likewise a run is a succession of idle states without any occupied slot so that a run is a period within which a secondary user could access the resource. Furthermore, if we assume that the longer the observation time the better the performance of the detection we understand that we are rather interested in runs above a given length or the complementary cumulated density function (CCDF) :

$$
\operatorname{Pr}(\text { run length } \geq k)=\left(1-p_{2}\right)^{k-1}
$$

It turns out that the runs have a geometric distribution, as observed in [7].

\section{IMPERFECT DETECTION}

Section 2 addressed only the true activity of the primary user (that is to say whether there is a secondary user or not). We now introduce an observer (the secondary user who wants to access the channel) which listens to a potential user already transmitting in the frequency band under consideration. The SU performs a detection, whatever it may be, and we only assume we know the two error probabilities of interest in our problem: the probability of non detection $\left(P_{N D}\right)$ and of false alarm $\left(P_{F A}\right)^{1}$.

From the SU point of view two metrics are of interest when assessing the quality of the opportunistic access to the spectrum:

- what is the collision rate with the primary user. It should be the least possible because the secondary user is not expected to create harmful interference to the primary user; it is a constraint we can express as a collision probability $p_{\text {coll }}$.

${ }^{1}$ These probabilities do depend, of course, on the detection scheme (matched filter, energy detection, ....) and on some parameters like SNR, number of samples and threshold used to take the decision, ... 
- what can the secondary user expect as succesful transmission in the channel, expressed as a probability $p_{\text {succ }}$ of successful access to the resource.

These two metrics are in fact the conditional probabilities of SU transmission to the true channel state:

$$
\begin{aligned}
p_{\text {coll }} & =\operatorname{Pr}(\text { transmit } \mid \text { channel is On }) \\
p_{\text {succ }} & =\operatorname{Pr}(\text { transmit } \mid \text { channel is Off })
\end{aligned}
$$

$p_{\text {coll }}$ and $p_{\text {succ }}$ depend obviously on the quality of the detection, but we introduce the possibility for the secondary user to discard the indication of the sensing. Indeed SU should not always obey to the information given by its detection scheme because it knows that the detector is prone, say, to non detecting existing signals; it is then envisionable not obeying systematically to the no signal present information, a kind of prudent (or conservative) policy. Likewise, when the detection scheme is prone to false alarm we can envision a more aggressive policy (sometimes we decide to use the resource even if the detector says that a signal is present, especially if we can afford for some collisions). Such policies are introduced in [2]. Here, they are taken into account by introducing a probability $\pi_{1}$ to obey the indication of the detection when the result is on, and a probability $\pi_{2}$ to obey the result of the detection when the result is off, as depicted in fig. 6 .

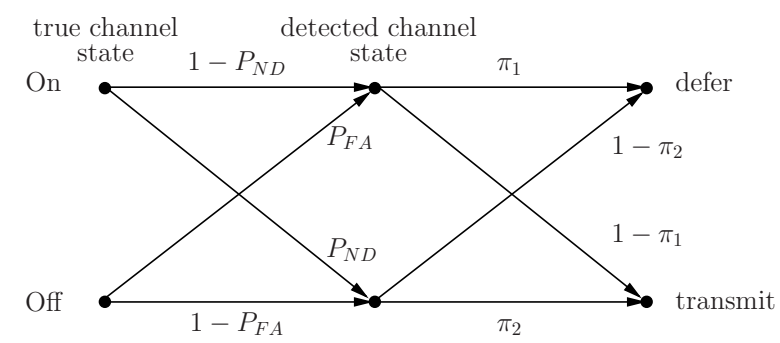

Figure 6: Introducing the possibility of disobeying the sensing

The collision and successful access probabilities can now easily be conputed:

$$
\begin{aligned}
p_{\text {coll }} & =\pi_{2} P_{N D}+\left(1-\pi_{1}\right)\left(1-P_{N D}\right) \\
p_{\text {succ }} & =\pi_{2}\left(1-P_{F A}\right)+\left(1-\pi_{1}\right) P_{F A}
\end{aligned}
$$

Substituting $\pi_{1}=\pi_{2}=1$ in (8) we have, as expected, $p_{\text {coll }}=$ $P_{N D}$ and $p_{\text {succ }}=1-P_{F A}$. It is interesting to notice that $p_{\text {coll }}$ and $p_{\text {succ }}$ have the same variation with respect to $\pi_{1}$ (resp. $\pi_{2}$ ) so that we cannot hope reducing $p_{\text {coll }}$ and at the same time increasing $p_{\text {succ }}$ by a change of a single parameter $\pi_{i}, i=1,2$.

Equations (8) can be rewritten in a vector form making apparent that the set $\mathcal{D}$ of allowable couples $\left(p_{\text {succ }}, p_{\text {coll }}\right)$ is the interior of the parallelogram built on two vectors $\mathbf{U}, \mathbf{V}$ whose ends are respectively the functioning point $\left(P_{F A}, 1-\right.$ $P_{N D)}$ of the ROC curve and its mirror $\left(1-P_{F A}, P_{N D}\right)$ as depicted in fig. 7:

$$
\begin{aligned}
\left(\begin{array}{c}
p_{\text {succ }} \\
p_{\text {coll }}
\end{array}\right) & =\left(1-\pi_{1}\right)\left(\begin{array}{c}
P_{F A} \\
1-P_{N D}
\end{array}\right)+\pi_{2}\left(\begin{array}{c}
1-P_{F A} \\
P_{N D}
\end{array}\right) \\
& =\left(1-\pi_{1}\right) \mathbf{U}+\pi_{2} \mathbf{V}
\end{aligned}
$$

The lower boundary of the parallelogram is the minimum

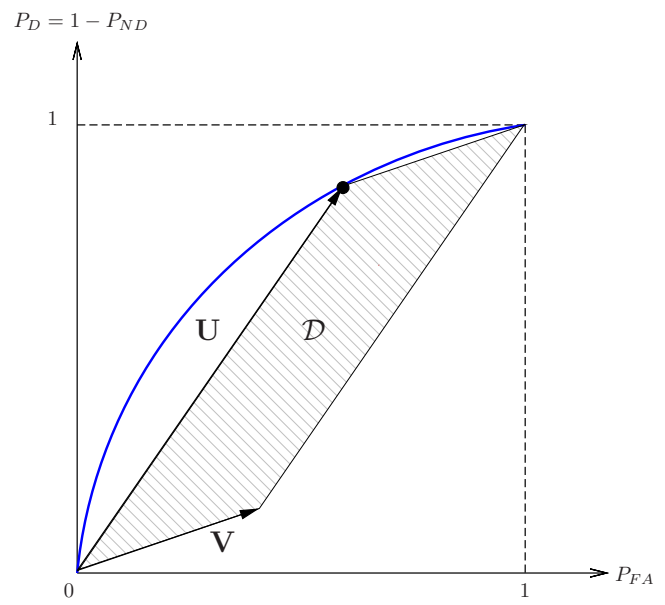

Figure 7: The set $\mathcal{D}$ of possible probabilities $p_{\text {succ }}$ and $p_{\text {coll }}$

collision probability for a given successful access probability, it corresponds to the two possible choices for the pair $\left(\pi_{1}, \pi_{2}\right): \pi_{1}=1, \pi_{2} \in[0,1]$ or $\pi_{1} \in[0,1], \pi_{2}=1 \mathrm{It}$ means that one of the results of the detection stage is always obeyed.

\section{DISTRIBUTION OF COLLISIONS AND SUCCESSFUL ACCESSES}

We now turn to the computation of the joint probability $\mathcal{A}_{n}(i, j)$ of having of $i$ successful access to the band and $j$ collisions among $n$ time slots. The quantities $\mathcal{A}_{n}(i, j)$ can be seen as the coefficients of a two variable polynomial

$$
P_{n}(Y, Z)=\sum_{i, j=0}^{n} \mathcal{A}_{n}(i, j) Y^{i} Z^{j}
$$

From (7) a collision occurs only when the true channel state is On and a successful access occurs only when the true channel state is Off. If we have $m$ occupied time-slots the distribution of the number of collisions is given by the coefficients of the polynomial $\left(1-p_{\text {coll }}+p_{\text {coll }} Y\right)^{m}$. Likewise, for the remaining $n-m$ time-slots (they are free), the number of successful access to the channel is given by the coefficients of the polynomial $\left(1-p_{\text {succ }}+p_{\text {succ }} Z\right)^{n-m}$.

As a result this computation involves the distribution $P(m, n)$ of the number of On states in $n$ times slots, already defined above in (4).

$$
\begin{aligned}
P_{n}(Y, Z)=\sum_{m=0}^{n} P(m, n) & \times\left(1-p_{\text {coll }}+p_{\text {coll }} Y\right)^{m} \\
\times & \left(1-p_{\text {succ }}+p_{\text {succ }} Z\right)^{n-m}
\end{aligned}
$$

We give below some examples of surfaces depicting $\mathcal{A}_{n}(i, j)$ for the same channel than section 3.1, and a functioning point moving on the ROC curve; for the sake of brevity and simplicity we suppose $\pi_{1}=\pi_{2}=1$ and we consider 3 values of the false alarm probability: $P_{F A}=0.05,0.2$, and 0.6 . 

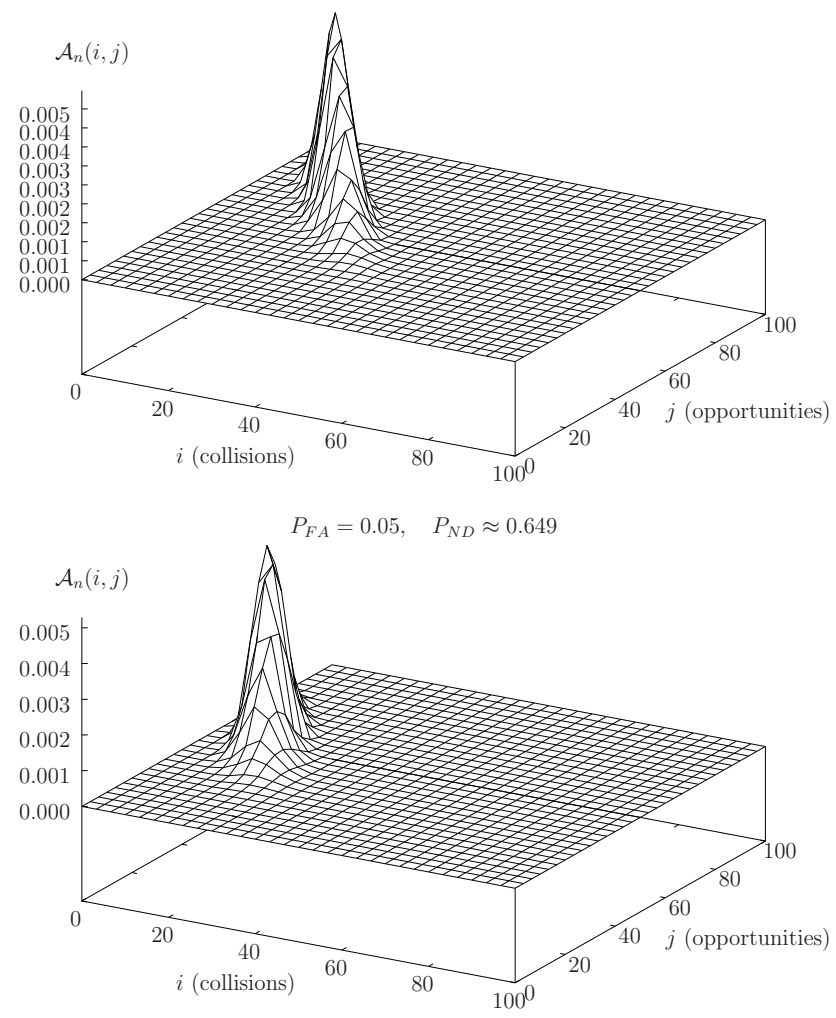

reduces to

$P_{n}(Y, Z)=\sum_{m=0}^{n} P(m, n)=1 \Rightarrow \mathcal{A}_{n}(i, j)= \begin{cases}1 & \text { if } i=j=0 \\ 0 & \text { otherwise }\end{cases}$

Likewise, when $p_{\text {coll }}=p_{\text {succ }}=1$ the $\mathrm{SU}$ always accesses the channel whatever the sensing result $\left(\pi_{1}=0\right.$ and $\left.\pi_{2}=1\right)$, so that it does not miss any opportunity but a the expense of a collision whenever the PU is present in the channel. The two variables enumerator reduces then to

$$
\begin{aligned}
& P_{n}(Y, Z)=\sum_{m=0}^{n} P(m, n) Y^{m} Z^{n-m} \\
& \quad \Rightarrow \mathcal{A}_{n}(i, j)= \begin{cases}0 & \text { if } j \neq n-i \\
P(i, n) & \text { if } j=n-i\end{cases}
\end{aligned}
$$

\subsubsection{Ideal detector}

The ideal detector corresponds to the case $P_{F A}=P_{N D}=0$ which ensures $p_{\text {coll }}=0$ and $p_{\text {succ }}=1$. With these values the two-variables enumerator is simplified and given by

$$
P_{n}(Y, Z)=\sum_{m=0}^{n} P(m, n) Z^{n-m}
$$

In this ideal case there is no collision at all ,so that $\mathcal{A}_{n}(i, j)=$ 0 when $i \neq 0$, and the distribution of successful access to the channel, given by $\mathcal{A}_{n}(0, j)=P(n-j, n)$, is given by the distribution of empty time-slots.

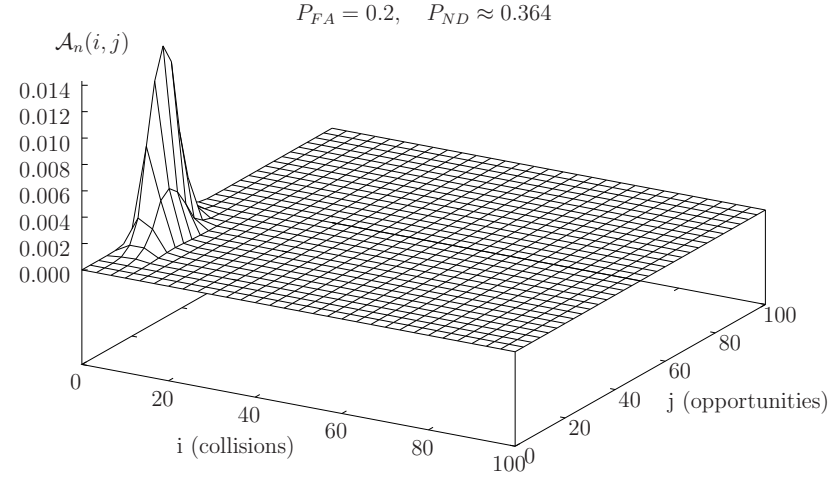

$P_{F A}=0.6, \quad P_{N D} \approx 0.090$

Figure 8: Surfaces for $P_{F A}=0.05,0.2,0.6$

As expected we observe that as $P_{F A}$ increases the number of collisions decreases (because $P_{N D}$ get smaller) but the number of successful opportunities is also reduced. So we see that the choice of an operating point on the ROC curve is the results of a trade-off. We observe that for small values of $P_{F A}$ the surface is close to the diagonal $i+j=n$, we give an explanation of this property in the next section.

\subsection{Special cases}

\subsubsection{Extreme points of $\mathcal{D}$}

The two points $(0,0)$ and $(1,1)$ are always in $\mathcal{D}$. When $p_{\text {coll }}=p_{\text {succ }}=0$ the SU never accesses the channel whatever the detection result $\left(\pi_{1}=1\right.$ and $\left.\pi_{2}=0\right)$ so it never creates collision with the PU but has no succesful access; (12) then

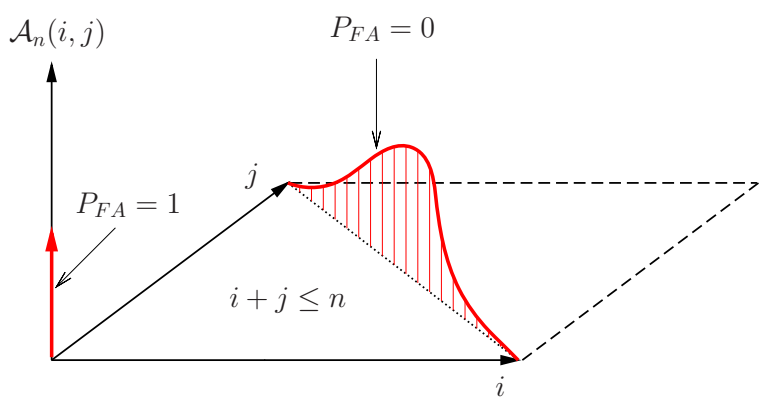

Figure 9: Special cases

\subsection{Marginal distributions}

They are obtained very simply by substituting $Y=1$ or $Z=1$ in (12). For instance:

$$
\begin{aligned}
P_{n}(Y, Z=1) & =\sum_{m=0}^{n} P(m, n) \times\left(1-p_{\text {coll }}+p_{\text {coll }} Y\right)^{m} \\
& =\sum_{i=0}^{n}\left\{\sum_{j=0}^{n} \mathcal{A}_{n}(i, j)\right\} Y^{i}
\end{aligned}
$$

The conditional distribution of the number of successful access with respect to the number of erasures is then given by

$$
\mathcal{A}_{n}(j \mid i)=\frac{\mathcal{A}_{n}(i, j)}{\sum_{j=0}^{n} \mathcal{A}_{n}(i, j)}
$$




\section{APPLICATION}

We now give a short example to show how the $\mathcal{A}_{n}(i, j)$ can be used in a realistic case. Erasure correcting codes for opportunistic spectrum access were introduced in $[10,11,12]$ as a solution to recover packets lost in collisions, especially LT codes [13]. Their main property is to allow the recovery of a complete length $n$ codeword provided a sufficient number of bits are correctly (i.e. without error) received. The design of such a code, that is the trade-off between redundancy and efficiency, is based on the knowledge of the erasure distribution and probabilities. In our problem the erasures are caused by sensing errors and their rate must be kept under a given level to protect the primary user.

We can thus suppose that the secondary user has a number $\mathcal{J}$ of packets to transmit opportunistically in the channel during the next $n$ time slots, with the constraint that the rate of collision is less than a defined threshold $\epsilon$, the number $\mathcal{J}$ being determined from the erasure recovery capacity of the code. The probability of a successful transmission of these packets, taking account of the forbidden time-slots and of the packets that had to be retransmitted because of a collision, is then given by

$$
P_{O K}(\mathcal{J})=\sum_{\substack{0 \leq i \leq\lfloor\epsilon n\rfloor \\ j \geq \mathcal{J}}} \mathcal{A}_{n}(i, j)
$$

Fig. 10 depicts the behaviour of $P_{O K}(\mathcal{J})$ for the same set of values of $P_{F A}$ and an arbitrary value $\epsilon=0.1$; it turns out that the result is very sensitive to the false alarm probability: it is only possible to transmit no more than $\mathcal{J} \approx 10$ packets with high probability if the operating point is set to $P_{F A}=0.6$. For $P_{F A}=0.2$ it is only possible to transmit $\approx 40$ packets with a $40 \%$ probability. The next figure

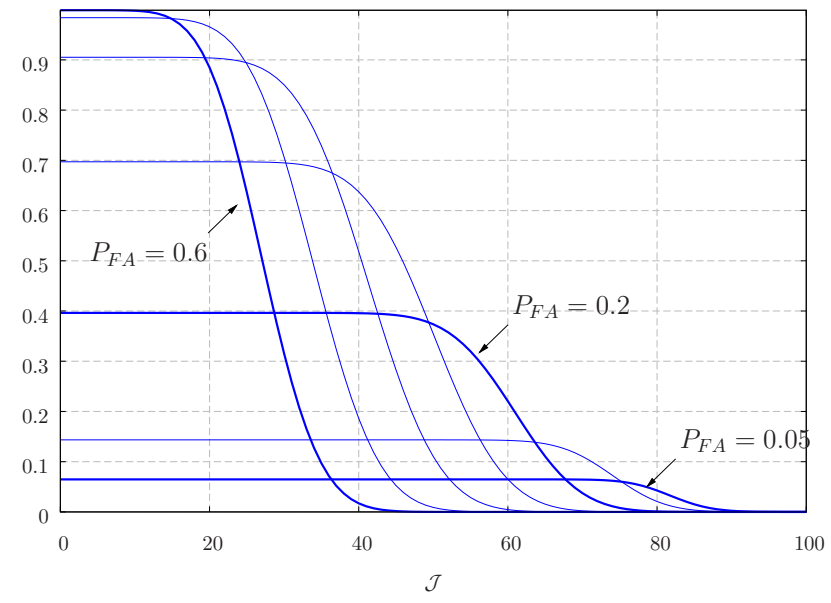

Figure 10: $P_{O K}$ for $P_{F A}=0.05,0.1,0.2,0.3,0.4,0.5,0.6$

(fig. 11) illustrates the behaviour of $P_{O K}$ for various values of the parameter $\epsilon$, and shows that increasing the allowed rate of collision from 0 to $10 \%$ helps in getting with a higher probability the same number of successful transmited packets (slightly greater than 10).

\section{CONCLUSION}

We have shown that we can separate the respective effects of the channel occupancy and of the errors of detection to

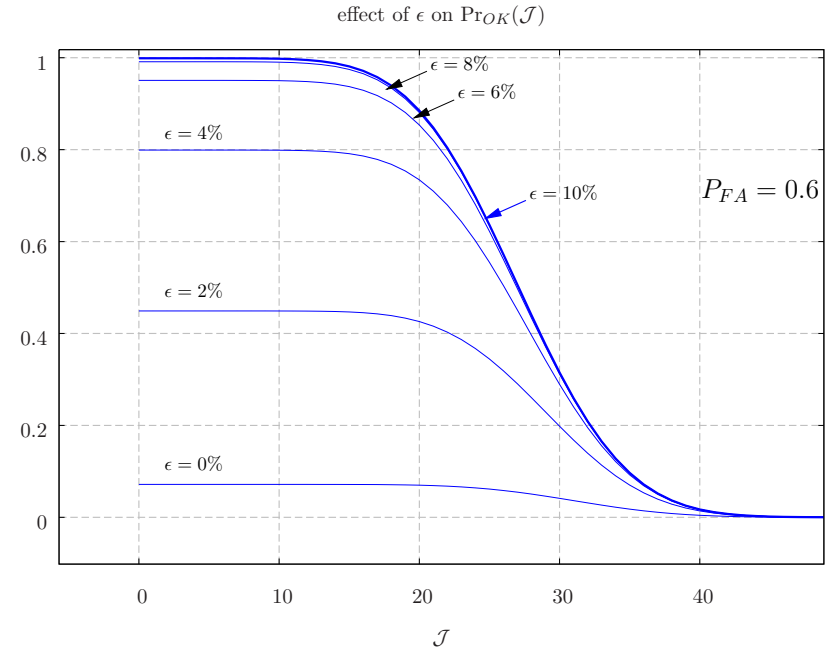

Figure 11: $P_{O K}$ for $P_{F A}=0.6$, effect of $\epsilon$

evaluate the performance of an opportunistic access to the spectrum which takes into account these two aspects of the problem. Channel occupancy behaviour is summarized in the distribution $P(m, n)$ of busy or idle time-slots and is easily computed with the help of two iterations. Sensing performance is given by the choice of an operating point on the ROC curve of the receiver. Finally, the global performance of the opportunistic access scheme is summarized in the coefficient of the two variable polynomial $P_{n}(Y, Z)$ whose computation is easy to implement and involves only the $P(m, n)$ and the two values $P_{F A}, P_{N D}$.

We did not assume that the SU has an estimation of the On/Off transition probabilities $p_{1}$ and $p_{2}$ of the PU's activity. We can expect that such an information would improve the performance of the opportunistic access to the channel because the SU could now take into account the conditional probability of the presence of the PU in the next time-slot in his decision to access or not the channel and thus avoid some collisions.

\section{ACKNOWLEDGEMENTS}

This work was supported by the European Commission in the framework of the FP7 FARAMIR Project.

\section{REFERENCES}

[1] M. Buddhikot, "Understanding dynamic spectrum access: Models, taxonomy and challenges," in Proceedings of IEEE DySPAN 2007, Dublin, Ireland, April 17-21 2007.

[2] Q. Zhao and B. Sadler, "A survey of dynamic spectrum access, signal processing, networking, and regulatory policy." IEEE Signal Processing Magazine, vol. 24, no. 3, pp. 79-89, may 2007.

[3] T. Yücek and H. Arslan, "A survey of spectrum sensing algorithms for cognitive radio applications," IEEE Communications surveys and tutorials, vol. 11, no. 1, pp. 116-130, 2009.

[4] D. Noguet and al. (2009) Sensing techniques for cognitive radio - state of the art and trends, edited by dominique noguet. IEEE SCC 41. 
[5] S. Haykin, D. Thomson, and J. Reed, "Spectrum sensing for cognitive radio," Proceedings of the IEEE, vol. vol. 97, no. 5, pp. 849-877, may 2009.

[6] T. Fawcett, "An introduction to ROC analysis," Pattern recognition letters, vol. 27, pp. 861-874, 2006.

[7] M. Wellens, J. Riihijärvi, and P. Mähönen, "Empirical time and frequency domain models of spectrum use," Elsevier Physical Communications, vol. 2, no. 1-2, pp. 10-32, 2009.

[8] E. Elliott, "Estimates of error rates for codes on burst noise channels," BSTJ, vol. 42, pp. 1977-1997, september 1963.

[9] L. Kanal and A. Sastry, "Models for channels with memory and their applications to error control," Proc. of the IEEE, vol. 66, no. 7, pp. 724-744, july 1978.

[10] H. Kushwaha and R. Chandramouli, "Secondary spectrum access with lt codes for delay-constrained applications," in proc. IEEE Consumer Communications and Networking conference (CCNC), Las Vegas, 2007.

[11] H. Kushwaha, Y. Xing, R. Chandramouli, and H. Heffes, "Reliable multimedia transmission over cognitive radio networks using [fountain codes."

[12] H. Kushwaha, Y. Xing, R. Chandramouli, and K. Subbalakshmi, Cognitive Networks: Towards Self-Aware Networks. Wiley, 2007, ch. Erasure tolerant coding for cognitive radios.

[13] M. Luby, "LT codes," in Foundations of Computer Science, 2002. Proceedings. The 43rd Annual IEEE Symposium on, 2002, pp. 271-280.

\section{APPENDIX}

The area $S(\mathcal{D})$ of the domain of possible values of $p_{\text {coll }}$ and $p_{\text {succ }}$ is given by the vector product of the vectors $\mathbf{V}$ and $\mathbf{U}$, that is, after a simple computation:

$$
S(\mathcal{D})=1-\left(P_{F A}+P_{N D}\right)
$$

This quantity is clearly nonnegative if we remember that the functioning point $\left(P_{F A}, 1-P_{N D}\right)$ on the ROC curve is above the diagonal $y=x$, so that $1-P_{N D} \geq P_{F A}$. Maximizing $S(\mathcal{D})$ is the equivalent to minimizing simultaneously $P_{F A}$ and $P_{N D}$; when these two probabilities are not independant but constrained by the ROC curve equation $1-P_{N D}=f\left(P_{F A}\right)$ we have

$$
S(\mathcal{D})=f\left(P_{F A}\right)-P_{F A}
$$

The extremal (in fact maximum) value of $S(\mathcal{D})$ is then reached for a false alarm probability $P_{F A}=p_{*}$ where the slope of the ROC curve is equal to 1 (see fig. 12).

This value $p_{*}$ yields the optimum functioning point of the ROC point, provided we give the same weight to $P_{F A}$ and $P_{N D}$. If not we can easily generalize by maximizing the suitably modified criterion:

$$
\begin{aligned}
\text { crit } & =\alpha\left(1-P_{F A}\right)+\beta\left(1-P_{N D}\right) \\
0 & \leq \alpha, \beta \leq 1, \quad \alpha+\beta=1
\end{aligned}
$$

When the operating point moves on the ROC curve it takes the form

$$
\text { crit }=\beta f\left(P_{F A}\right)+\alpha\left(1-P_{F A}\right)
$$

and its derivative with respect to $P_{F A}$ is zero when $\beta f^{\prime}\left(P_{F A}\right)-$ $\alpha=0$, that is to say on the operating poing where the tan-

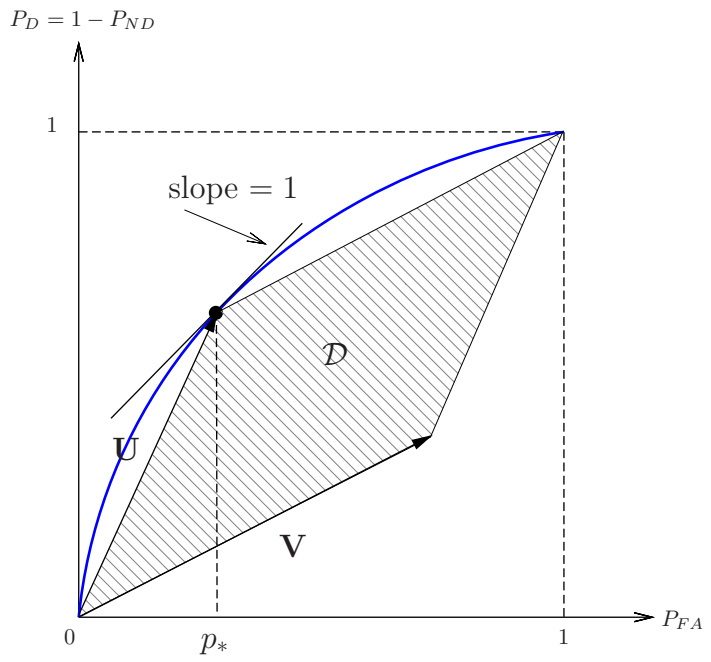

Figure 12: Optimum operating point minimizing $P_{F A}+P_{N D}$

gent to the ROC curve is equal to $\alpha / \beta$. For $\alpha>\beta$ this optimum operating point lies in the region $P_{F A}<p_{*}$ which is the region where the non detection probability grows faster than $P_{F A}$. Conversely, for $\alpha<\beta$ the operating point is in the region $P_{F A}>p_{*}$ where the false alarm probability grows faster than $P_{N D}$. 